\title{
Escala de avaliação das estratégias de regulação da motivação de alunos universitários: um estudo piloto
}

\section{Motivacional regulation strategies scale for college students: a pilot study}

\author{
Natália Moraes Góes, Evely Boruchovitch \\ Universidade Estadual de Campinas, Brasil
}

\begin{abstract}
Resumo
Os objetivos do estudo foram: a) realizar um estudo piloto para verificar a adequação da tradução de uma escala que mensura o uso de estratégias de regulação da motivação de estudantes universitários, originalmente da língua inglesa (Wolters \& Benzon, 2013), para a língua portuguesa e, b) analisar o autorrelato do uso dessas estratégias em função do sexo e área de conhecimento do curso dos estudantes. Participaram 42 estudantes de cursos de licenciaturas diversas que responderam a versão traduzida da escala e uma questão sobre a compreensão dos itens. Resultados revelaram que a escala se mostrou adequada para uso no Brasil.

Palavras-chave: estratégias de regulação da motivação, alunos universitários, autorregulação
\end{abstract}

\begin{abstract}
The objectives of the study were: a) to carry out a pilot study to evaluate the adequacy of a translation of an English written scale aimed at assessing motivational regulation strategies of college students to portuguese language and b) to exam the self-report of use of these strategies among college students in relation to participants'sex and course area. The sample was composed of 42 college students from different course areas who responded to the translated version of the scale and question about their comprehension of the items. Results showed that the scale was adequate to its use in Brazil.
\end{abstract}

Keywords: Motivational regulation strategies, college students, self-regulation

Habilidades autorregulatórias são primordiais para o sucesso escolar. Zimmerman (1990, 2002) afirma que a autorregulação não é uma habilidade mental ou uma habilidade de desempenho acadêmico. Mais precisamente, a autorregulação se refere ao autogerenciamento do pensamento, sentimentos e comportamentos orientados para atingir uma determinada meta (Zimmerman, 1990, 2002).

Um dos modelos mais referenciados atualmente sobre a autorregulação é o de Zimmerman (1998). O teórico propôs um modelo cíclico de autorregulação composto por três fases, a saber: planejamento, execução e autoavaliação.

A fase do planejamento consiste em estabelecer objetivos, selecionar as estratégias mais adequadas considerando as características da tarefa e os objetivos, avaliar a autoeficácia, crenças e interesses intrínsecos pela tarefa e a orientação para metas.

No momento da execução, os estudantes empregam as estratégias de aprendizagem com a finalidade de atingir os objetivos estabelecidos e a realização da tarefa. Para isso, fazem o uso do monitoramento e do controle das estratégias, da aprendizagem e da própria motivação.

$\mathrm{Na}$ autoavaliação, o estudante examina se deve prosseguir com novas aprendizagens ou retomar aspectos do conteúdo que não foram aprendidos (Zimmerman, 2002; Bembennuty, 2015).

Tal como os alunos podem regular a sua aprendizagem, eles também são capazes de regular a própria motivação. A autorregulação da motivação é considerada, atualmente, essencial no âmbito da autorregulação da aprendizagem (Paulino \& Silva, 2012, Paulino, Sá \& Silva, 2015).

A regulação da motivação caracteriza-se como um tipo de autocontrole que o estudante exerce sobre a sua própria motivação. Este constructo tem sido incorporado em diferentes modelos de autorregulação, incluindo aqueles que enfatizam a volição, que a define como uma habilidade individual que assegura a realização de atividades e metas, mesmo diante de distratores e atividades mais prazerosas (Wolters, 1999; Wolters \& Rosenthal, 2000).

Wolters (1999) e Wolters e Rosenthal (2000) identificaram cinco estratégias de regulação da motivação mais utilizadas por alunos do Ensino Fundamental, Médio e Superior de diferentes países: estratégias de autoconsequenciação, controle do ambiente, aumento do interesse para aprender, conversa consigo mesmo sobre o seu desempenho escolar e conversa consigo mesmo sobre a sua capacidade para aprender.

As estratégias de autoconsequenciação estão relacionadas a recompensas extrínsecas autoatribuídas pelos estudantes, se realizarem ou finalizarem a tarefa escolar. Já as estratégias de controle do ambiente referem ao esforço do estudante em concentrar sua atenção na tarefa, reduzindo as distrações do ambiente e/ou até mesmo organizando o ambiente de estudo para completar as tarefas. A estratégia de aumento do interesse para aprender consiste no trabalho dos estudantes para aumentar o seu esforço ou tempo na tarefa, ao tentarem 
torná-las mais agradáveis ou interessantes. A estratégia de conversar consigo mesmo sobre o seu desempenho escolar refere-se ao pensamento dos alunos em aumentar a sua vontade para completar a tarefa, intensificando seu foco no objetivo de obter boas notas. Por fim, a estratégia conversa consigo mesmo sobre a sua capacidade para aprender diz respeito à habilidade do aluno em tornar a tarefa mais relevante para ser realizada.

Em pesquisa realizada mais recentemente com alunos universitários, Wolters e Benzon (2013) identificaram mais um tipo de estratégia de regulação da motivação, as de regulação do valor.

A escassez de estudos nacionais que investigam a regulação da motivação, a ausência de instrumentos brasileiros que mensure o uso dessas estratégias, bem como a importância de realizar pesquisas transculturais, possibilitadas pela tradução e adaptação de escalas nortearam a realização do presente estudo. Assim, seus objetivos foram: a) realizar um estudo piloto para verificar a adequação da tradução de uma escala que mensura o uso de estratégias de regulação da motivação de estudantes universitários, originalmente da língua inglesa (Wolters \& Benzon, 2013), para a língua portuguesa e, b) analisar o autorrelato do uso dessas estratégias em função do sexo e do curso dos estudantes.

\section{Método}

\section{Participantes}

Participaram do estudo 42 estudantes de cursos de licenciaturas diversas de uma universidade pública localizada no interior do Estado de São Paulo/Brasil. Destes, 13 (30,9\%) estavam se graduando em cursos da área de Ciências Biológicas, $11(26,2 \%)$ na área de Ciências Exatas e 18 (42,8\%) na área de Ciências Humanas. Dos participantes, $27(64,3 \%)$ eram do sexo feminino e $15(35,7 \%)$ do sexo masculino.

\section{Instrumento}

Utilizou-se no presente estudo a Escala de avaliação das estratégias de regulação da motivação para estudantes universitários, desenvolvida e validada por Wolters e Benzon (2013), após permissão dos autores.

A escala tem como objetivo conhecer as diferentes estratégias de regulação da motivação utilizadas por estudantes universitários para sustentar ou manter seu esforço, persistência ou desejo de completar uma tarefa acadêmica. É de tipo Likert,composta por 30 itens e com 7 opções de respostas, variando de 1 - de forma alguma até 7 - muito frequentemente.

Os itens foram organizados em 6 fatores: regulação do valor $(\alpha=.91)$, regulação do desempenho $(\alpha=.84)$, autoconsequenciação $(\alpha=.91)$, controle do ambiente $(\alpha=.77)$, regulação do interesse situacional $(\alpha=.88)$ e regulação da meta para aprender $(\alpha=.88)$.

O Fator 1 - Regulação do valor é composto por 6 itens $(1,2,3,4,5,6)$ e se refere ao esforço dos estudantes em tornar o conteúdo mais interessante, útil ou importante para aprender. Como exemplo de item do Fator 1 pode-se apontar: "Faço um esforço para relacionar o que estamos aprendendo aos meus interesses pessoais".
O Fator 2 - Regulação do desempenho contêm 5 itens $(7,8,9,10,11)$ e diz respeito a crença dos estudantes em fazer um bom trabalho ou tirar boas notas nas atividades que eles precisam desenvolver $(\alpha=84)$. Como exemplo de item do Fator 2, pode-se citar:"Lembro a mim mesmo o quanto é importante tirar boas notas".

O Fator 3 - Autoconsequenciação composto por 5 itens $(12,13,14,15,16)$ consiste em o estudante se autorrecompensar ao finalizar ou realizar uma tarefa $(\alpha=.91)$. Como exemplo de item do Fator 3 pode-se mencionar:"Prometo a mim mesmo algum tipo de recompensa, se eu terminar minhas leituras".

$\mathrm{O}$ Fator 4 - Controle do ambiente com 4 itens $(17,18,19,20)$ faz referência ao esforço do estudante em controlar o seu espaço físico com a finalidade de realizar a tarefa prescrita $(\alpha=.77)$. Como exemplo de item do Fator 4: "Modifico o ambiente ao meu redor de forma que fique mais fácil me concentrar nas tarefas escolares".

O Fator 5 - Regulação do interesse situacional composto por 5 itens $(21,22,23,24,25)$ representa estratégias que tentam deixar os estudos mais agradáveis e divertidos $(\alpha=88)$. Como exemplo de item do Fator 5 pode-se apontar: "Tento mostrar a mim mesmo que realizar as atividades escolares pode ser divertido".

O Fator 6 - Regulação da meta para aprender composto por 5 itens $(26,27,28,29,30)$ consiste no esforço do estudante para realçar o seu desejo de melhorar a sua compreensão ou aprender o máximo que puder $(\alpha=.88)$. Como exemplo de item do Fator 6 pode-se citar: "Desafio a mim mesmo a terminar as tarefas escolares e aprender o máximo possível".

Para atender os objetivos do presente estudo, foi incluída, no final da escala, uma questão que visava avaliar a opinião dos participantes acerca da clareza de redação dos itens da escala, bem como se tinham alguma sugestão para o aprimoramento do instrumento.

\section{Procedimentos}

A escala foi aplicada pela primeira no Brasil, após alguns procedimentos de tradução terem sido realizados.

Inicialmente a primeira autora do presente estudo verificou quais itens eram semelhantes aos da "Escala de avaliação das estratégias de regulação da motivação para alunos do Ensino Médio", instrumento desenvolvido pelo mesmo autor (Wolters, 1999), já traduzida para o português por Boruchovitch e Felicori (2015), ambas conhecedoras da temática do instrumento e com domínio da língua inglesa. Os itens diferentes foram então traduzidos pela primeira autora do presente estudo. A revisão da tradução desses itens foi realizada por Boruchovitch e Felicori. Realizados os ajustes necessários, a escala foi enviada para um tradutor para fazer a back-translation. Após o recebimento da versão referente ao back-translation, uma nova análise dos itens foi realizada. Algumas discrepâncias foram encontradas em 7 itens que tiveram sua tradução revisada e reenviada, novamente para back-translation. Depois desses cuidados, a escala pareceu adequada para a realização de um estudo piloto com universitários brasileiros.

A coleta de dados se iniciou após a aprovação do trabalho pelo Comitê de Ética (CAE 64526117.3.0000.5404). 
Inicialmente foi realizado contato com três professores das disciplinas de Estágio Supervisionado de uma universidade pública localizada no interior do Estado de SãoPaulo/Brasil, para que autorizassem a coleta e indicassem o melhor momento para que ela acontecesse. A disciplina de Estágio Supervisionado foi escolhida, pelo fato dos alunos matriculados serem de cursos e áreas do conhecimento diversas. As coletas ocorreram presencialmente, no mês de Março de 2017.

Durante a coleta, os objetivos da pesquisa foram explicados e os estudantes informados da ausência de qualquer prejuízo em decorrência da não participação. Chamou-se a atenção para a questão final, em que foi pedido aos alunos que expressassem sua opinião sobre a sua compreensão acerca da clareza dos itens da Escala. $\mathrm{Na}$ sequência, os alunos receberam o Termo de Consentimento Livre e Esclarecido (TCLE), mediante a assinatura do documento, a Escala foi entregue. A coleta de dados transcorreu tranquilamente, não tendo surgido qualquer dúvida ou questionamento por parte da amostra. Durou aproximadamente 20 minutos.

\section{Análise dos Dados}

Os dados quantitativos foram analisados utilizando o software SPSS versão 20.0. Inicialmente calculou-se a consistência interna da escala. Na sequência, foram estimados os valores totais da escala, as médias por fatores, por itens e os desvios-padrões. Realizou-se o teste de normalidade dos itens da escala, constatando-se que os dados não apresentaram uma distribuição normal. Diante disso foram realizados os testes não-paramétricos Mann Whitney para verificar se haviam diferenças estatisticamente significantes entre as estratégias de regulação motivacional e o sexo e o teste Kruskal Wallis para averiguar se haviam diferenças entre as áreas de conhecimento e as estratégias de regulação motivacional. A questão aberta foi examinada por meio da análise de conteúdo (Bardin, 2004).

\section{Resultados}

Com a finalidade de atingir o primeiro objetivo do estudo foram analisadas as respostas dos alunos à questão aberta: "Você teve dúvidas em relação a algum item da Escala? Em caso afirmativo, relate-as. O seu relato é muito importante para que possamos refinar o instrumento".

Verificou-se que, 34 estudantes $(80,9 \%)$ não apresentaram dúvidas durante o preenchimento da Escala. No entanto, $8(19,0 \%)$ as tiveram e ofereceram sugestões para adequá-la. Entre os problemas apontados pela minoria, houve dificuldade de perceber diferenças de conteúdo dos itens 12 ao 16 e 27 ao 30, como pode ser visto no relato de um dos estudantes: "Muitos itens pareciam exatamente iguais para mim. Pensei em uma mesma situação quando os li, fazendo com que os avaliasse de maneira igual". Um estudante indicou dificuldades em relacionar o conteúdo das estratégias tratadas nos itens 12 ao 17 à sua vida universitária e um estudante indicou que o termo "apenas" utilizado em um dos itens limitou a sua resposta.
Como as respostas dessa minoria de estudantes à questão aberta não indicaram problemas na compreensão dos itens, não foram feitas alterações na escala.

Para examinar a consistência interna da escala calculou-se o alpha de Cronbach obtendo-se um alpha de 0,90 para escala total o que indica um alto índice de consistência interna. Os fatores também apresentaram bons índices de consistência interna: Fator 1-Regulação do valor $(\alpha=0,79)$, Fator 2 - Regulação do desempenho $(\alpha=0,88)$, Fator 3 - Autoconsequenciação $(\alpha=0,97)$, Fator 4 - Controle do ambiente $(\alpha=0,85)$, Fator 5 Regulação do interesse situacional $(\alpha=0,84)$ e Fator 6 Regulação da meta para aprender $(\alpha=0,92)$.

A Tabela 1 apresenta os dados obtidos a partir do autorrelato do uso das estratégias de regulação da motivação.

Tabela 1.

Médias, valores máximos e mínimos e desvio padrão por fator e por itens da Escala de regulação da motivação.

\begin{tabular}{|c|c|c|c|c|c|}
\hline Fatores & Itens & Mín & Máx & Média & $\mathrm{DP}$ \\
\hline \multirow{6}{*}{ Fator 1} & Item 1 & 3 & 7 & 5,62 & 1,15 \\
\hline & Item 2 & 1 & 7 & 5,79 & 1,55 \\
\hline & Item 3 & 3 & 7 & 5,76 & 1,16 \\
\hline & Item 4 & 3 & 7 & 5,67 & 1,18 \\
\hline & Item 5 & 1 & 7 & 4,67 & 2,02 \\
\hline & Item 6 & 1 & 7 & 4,93 & 1,80 \\
\hline Total Fator 1 & & 1 & 7 & 5,40 & 1,06 \\
\hline \multirow{5}{*}{ Fator 2} & Item 7 & 1 & 7 & 5,02 & 1,54 \\
\hline & Item 8 & 1 & 7 & 5,40 & 1,62 \\
\hline & Item 9 & 1 & 7 & 5,33 & 1,32 \\
\hline & Item 10 & 1 & 7 & 5,10 & 1,51 \\
\hline & Item 11 & 2 & 7 & 5,45 & 1,36 \\
\hline Total Fator 2 & & 1 & 7 & 5,26 & 1,21 \\
\hline \multirow{5}{*}{ Fator 3} & Item 12 & 1 & 7 & 3,38 & 2,02 \\
\hline & Item 13 & 1 & 7 & 3,64 & 2,23 \\
\hline & Item 14 & 1 & 7 & 3,81 & 2,04 \\
\hline & Item 15 & 1 & 7 & 3,60 & 2,11 \\
\hline & Item 16 & 1 & 7 & 3,40 & 1,99 \\
\hline Total Fator 3 & & 1 & 7 & 3,57 & 1,97 \\
\hline \multirow{4}{*}{ Fator 4} & Item 17 & 1 & 7 & 4,48 & 1,76 \\
\hline & Item 18 & 1 & 7 & 4,43 & 1,52 \\
\hline & Item 19 & 1 & 7 & 5,29 & 1,61 \\
\hline & Item 20 & 3 & 7 & 5,98 & 1,11 \\
\hline Total Fator 4 & & 1 & 7 & 5,04 & 1,27 \\
\hline \multirow{5}{*}{ Fator 5} & Item 21 & 1 & 7 & 2,33 & 1,68 \\
\hline & Item 22 & 1 & 7 & 2,45 & 1,67 \\
\hline & Item 23 & 1 & 7 & 3,67 & 1,86 \\
\hline & Item 24 & 1 & 7 & 3,88 & 1,67 \\
\hline & Item 25 & 1 & 7 & 4,24 & 1,72 \\
\hline Total Fator 5 & & 1 & 7 & 3,31 & 1,35 \\
\hline \multirow{5}{*}{ Fator 6} & Item 26 & 1 & 7 & 4,26 & 1,84 \\
\hline & Item 27 & 1 & 7 & 4,36 & 1,80 \\
\hline & Item 28 & 1 & 7 & 4,17 & 1,80 \\
\hline & Item 29 & 1 & 7 & 3,83 & 1,90 \\
\hline & Item 30 & 1 & 7 & 3,71 & 1,93 \\
\hline Total Fator 6 & & 1 & 7 & 4,07 & 1,64 \\
\hline
\end{tabular}


Como pode ser visto na Tabela 1 , os valores mínimos variaram de 1 a 3 e o valor máximo foi 7 para todos os itens. No que se refere ao desvio padrão verificou-se que a maior dispersão se deu no Fator 3 Autoconsequenciação $(\mathrm{DP}=1,97)$ e a menor no Fator 1 Regulação do valor ( $\mathrm{DP}=1,06)$.

A média mais alta por fator foi encontrada no Fator 1 Regulação do valor $(\mathrm{M}=5,40)$ e a mais baixa foi no fator 5 - Regulação do interesse situacional ( $\mathrm{M}=3,31)$.

A análise das médias por itens mostrou que no Fator 4 - Controle do ambiente, encontra-se o item com maior média, comparado a todos os outros da Escala, o item 20 - "Tento estudar num momento em que consigo ficar mais focado" (M=5,98) e no Fator 5 - Regulação do interesse situacional, o item com menor média, item 21 "Deixo o estudo mais agradável ao transformá-lo em um jogo" $(\mathrm{M}=2,33)$.

A Tabela 2 apresenta os resultados em função do sexo e área de conhecimento (cursos) dos participantes.

Tabela 2.

Médias por fator com base na área de conhecimento $e$ sexo dos participantes.

\begin{tabular}{cccc|cc}
\hline & \multicolumn{3}{c}{$\begin{array}{c}\text { Área de conhecimento } \\
\text { (cursos) }\end{array}$} \\
\cline { 2 - 6 } Fatores & $\begin{array}{c}\mathrm{CH} \\
(\mathrm{n}=18)\end{array}$ & $\begin{array}{c}\mathrm{CB} \\
(\mathrm{n}=13)\end{array}$ & $\begin{array}{c}\mathrm{CE} \\
(\mathrm{n}=11)\end{array}$ & $\begin{array}{c}\mathrm{F} \\
(\mathrm{n}=27)\end{array}$ & $\begin{array}{c}\mathrm{M} \\
(\mathrm{n}=15)\end{array}$ \\
\hline Fator 1 & 5,54 & 5,32 & 5,29 & 5,56 & 5,12 \\
Fator 2 & 5,33 & 4,77 & 5,73 & 5,47 & 4,90 \\
Fator 3 & 3,57 & 3,77 & 3,33 & 4,18 & 2,45 \\
Fator 4 & 5,05 & 4,58 & 5,57 & 4,91 & 5,28 \\
Fator 5 & 3,33 & 3,58 & 2,96 & 3,37 & 3,21 \\
Fator 6 & 4,33 & 3,46 & 4,34 & 3,81 & 4,52 \\
\hline Média Total & 4,54 & 4,27 & 4,53 & 4,57 & 4,24 \\
\hline
\end{tabular}

Legenda: $\mathrm{CH}=$ Ciências Humanas, $\mathrm{CE}=\mathrm{Ciências} \mathrm{Exatas} \mathrm{e} \mathrm{CB}=$ Ciências Biológicas.

Como pode ser visto na Tabela 2, as médias por área de conhecimento foram bem próximas (Ciências Humanas, $M=4,54$; Ciências Biológicas, $M=4,27$ e Ciências Exatas, $M=4,53)$ tanto que não foram encontradas diferenças estatisticamente significantes entre elas $(\mathrm{p}<0,05)$.

Médias bem próximas foram localizadas também em função do sexo (Feminino, $M=4,57$ e Masculino, $\mathrm{M}=4,24)$ o que não suscitou diferenças estatisticamente significantes. No entanto, para esta variável foi encontrada uma diferença estatisticamente significante apenas no fator 3 - Autoconsequenciação. As estudantes do sexo feminino $(\mathrm{M}=4,18)$ reportaram utilizar significativamente mais essas estratégias do que os do sexo masculino $(M=2,45)$.

\section{Discussão}

O primeiro objetivo do presente estudo foi verificar a adequação da tradução de uma escala sobre estratégias de regulação da motivação, originalmente da língua inglesa, para a língua portuguesa para uso entre universitários brasileiros. O estudo piloto permitiu constatar que a escala parece estar adequada para ser aplicada em alunos desse segmento da escolarização. Ela parece examinar o que se propõe e apresentou alta consistência interna tanto no seu total como em cada um dos seus fatores. Além disso, foi possível constatar que a maioria dos participantes não teve dúvidas, ao preenchê-la. As poucas considerações feitas por uma minoria de alunos não indicou necessidade de qualquer reformulação. Entretanto, destaca-se que a amostra, ora empregada foi pequena e pouco representativa. Certamente, novas coletas em amostras maiores e mais representativas trarão novos insights para o aperfeiçoamento do instrumento em nosso meio.

Os resultados da presente pesquisa também revelaram, de modo geral, que os estudantes universitários que compuseram a amostra relatam utilizar moderadamente estratégias de regulação da motivação.

Todavia, algumas estratégias apresentaram médias mais altas do que outras. Constatou-se que as estratégias que compõem o fator Regulação do valor foram as mais indicadas no presente estudo. Este resultado diverge dos encontrados por Schwinger e Otterpohl (2017) e Wolters e Benzon (2013) que verificaram que as estratégias de autoconsequenciação apresentaram as maiores médias em uma amostra de 531 estudantes do Ensino Médio e 215 universitários respectivamente. As estratégias com menor média foram as de regulação do interesse situacional, como constatado em estudo anterior (Schwinger \& Otterpohl, 2017).

As médias por fatores também revelaram que os participantes apresentam grande preocupação em evitar tirar notas baixas. Esse fato pode ser visto pelo fator Regulação do desempenho ter apresentado uma das maiores médias. Paulino, Sá e Silva (2015) também averiguaram essa tendência em estudo anterior, em que os alunos obtiveram maiores médias para as estratégias de regulação do desempenho em uma amostra composta por 316 estudantes do $7^{\circ}$ ao $9^{\circ}$ ano do Ensino Fundamental.

Wolters e Benzon (2013) verificaram que os estudantes, de modo geral, tendem a utilizar estratégias mais voltadas para a permanência do foco para o estudo. Esse dado converge com o encontrado no presente estudo, visto que, a média mais alta obtida na Escala foi na estratégia: "Tento estudar em um momento em que consigo ficar mais focado".

No que diz respeito aos itens mais e menos indicados na Escala, algumas hipóteses podem ser aventadas. Com relação ao item com menor média (item 21) pode-se supor que transformar o estudo em um jogo seja uma estratégia distante da realidade dos estudantes universitários. É possível que seja mais utilizada por alunos do Ensino Fundamental e Médio. O tempo exigido para utilizar esta estratégia também pode ser levado em consideração para o seu baixo relato de uso, o que levaria os estudantes a preferirem estratégias que demandem menos tempo, mas que tenham o mesmo efeito sobre a regulação da motivação. Outro fator que pode ter contribuído para essa média ter sido baixa é o desconhecimento da estratégia e dos seus benefícios.

A respeito do fato da estratégia mais apontada pelos alunos, item 20, pode-se supor que eles apresentam conhecimento metacognitivo, visto que buscam momentos em que estariam mais focados para estudar.

Em relação ao sexo dos participantes e o uso de estratégias de regulação da motivação, verificou-se 
diferença estatisticamente significativa apenas no Fator Autoconsequnciação, isto é, participantes do sexo feminino relatam utilizar mais estas estratégias do que os do sexo masculino. Diferente do presente estudo, Cooper e Corpus (2008) não encontraram diferenças estatisticamente significativas relacionadas ao sexo dos participantes. Em contrapartida, Paulino, Sá e Silva (2015) verificaram que o sexo feminino, de modo geral, utiliza mais estratégias de regulação da motivação do que o sexo masculino. Vale ressaltar que, no presente estudo a variável sexo não estava equilibrada, tendo a amostra sido composta de um número maior de participantes do sexo feminino o que, certamente, pode ter interferido nos resultados obtidos.

A área de conhecimento também não foi uma variável na qual foram encontradas diferenças estatisticamente significativas. Futuros estudos devem ser conduzidos para avaliar melhor o impacto do sexo e da área de conhecimento no relato de uso das estratégias de regulação da motivação.

\section{Considerações Finais}

Uma das características essenciais da autorregulação é a capacidade do estudante de controlar e manter a sua própria motivação (Schwinger \& Otterpohl, 2017). Conhecer e promover o uso de estratégias de regulação da motivação entre alunos independentemente do nível de escolarização é fundamental.

A tradução para a língua portuguesa de uma escala para avaliar a regulação da motivação de alunos universitários foi uma iniciativa importante já que inexistiam instrumentos brasileiros para mensurar essa variável tão relevante para o sucesso escolar e acadêmico. Espera-se que o presente estudo possa despertar interesse de outros pesquisadores e que o uso ampliado da escala possa viabilizar a realização de novas pesquisas que colaborem para o desenvolvimento de ações diagnósticas e interventivas orientadas ao fortalecimento da regulação motivacional de estudantes, sobretudo daqueles que desejam ser professores.

\section{Referências}

Bardin, L. (2004). .Análise de Conteúdo. 3.ed. Portugal: Edições 70.

Bembenutty, H. (2015). Self-regulated learning and development in teacher preparation training. Springer Briefs in Education, 9-28. https://link.springer.com/chapter/10.1007\%2F978-94017-9950-8_2

Boruchovitch, E. \& Felicori, C.M. (2015). Tradução de escala de estratégias de regulação da motivação de alunos do Ensino Médio. Manuscrito não publicado. Universidade Estadual de Campinas, Campinas, São Paulo, Brasil.

Cooper, C. \& Corpus, J.H. (2008). Learners' developing knowledge of strategies for regulation motivation. Journal of applied developmental psychology, 30 (4), 525-536.

Paulino, P. \& Silva, A.L. (2012). Promover a regulação da motivação na aprendizagem. Cadernos de Educação, https://periodicos.ufpel.edu.br/ojs2/index.php/caduc/a rticle/view/2150

Paulino, P. Sá, I. \& Silva, A.L. (2015). Autorregulação da motivação: crença e estratégias de alunos portugueses do $7^{\circ}$ ao $9^{\circ}$ ano de escolaridade. Psicologia Reflexão e Crítica, 28(3), 574-582. http://www.scielo.br/scielo.php?pid=S0102-79722015 $000300574 \&$ script $=$ sci_abstract\&tlng=pt

Schwinger, M. \& Otterpohl, N. (2017). Which one works best? Considering the relative importance of motivational regulation strategies. Learning and individual differences, 53, 122-132. http://www.sciencedirect.com/science/article/pii/S104 1608016302862

Wolters, C.A. (1999). The relation between high school students' motivational regulation and their use of learning strategies, effort, and classroom performance. Learning and Individual Differences, 3(3), 281-299. http://www.sciencedirect.com/science/article/pii/S104 1608099800041

Wolters, C. A. (2003). Conceptualizing the Role and Influence of Student- Teacher Relationships on Children's Social and Cognitive Development, 189-205.

Wolters, C.A. \& Benzon, M.B. (2013). Assessing and predicting college students'use of strategies for the self-regulation of motivation. The Journal of Experimental Education, 81 (2), 199-221. http://www.tandfonline.com/doi/abs/10.1080/0022097 3.2012.699901

Wolters, C.A. \& Rosenthal, H. (2000). The relation between student's motivational beliefs and their use of motivational regulation strategies. International Journal of Educational Research, 33, 801-820. http://www.sciencedirect.com/science/article/pii/S088 3035500000513

Zimmerman, B.J. (1990). Self-regulated learning and academic achievement: an overview. Education Psychologist, 25(1), 3-17. http://www.tandfonline.com/doi/abs/10.1207/s 153269 85ep2501_2

Zimmerman, B.J. (1998). Academic studying and the development of personal skill: a self-regulated perspective. Educational Psychologist, 33(2/3), 73-86.

Zimmerman, B.J. (2002). Becoming a self-regulated learner: an overview. Theory into practice, 41(2), 64-70.

\section{Agradecimentos}

As autoras agradecem o apoio financeiro da Capes e do CNPq. 